\title{
dspace.vutbr.cz
}

\section{Practical Design of Fractional-Order Oscillator Employing Simple Resonator and Negative Resistor}

ŠOTNER, R.; JEŘÁBEK, J.; DOMANSKÝ, O.; HERENCSÁR, N.; KARTCI, A.; DVOŘÁK, J.

Proceedings of 10th International Congress on Ultra Modern Telecommunications and Control Systems - ICUMT 2018

pp. 1-4

elSBN: 978-1-5386-9361-2

DOI: http://dx.doi.org/10.1109/ICUMT.2018.8631226

Accepted manuscript

(C2018 IEEE. Personal use of this material is permitted. Permission from IEEE must be obtained for all other uses, in any current or future media, including reprinting/republishing this material for advertising or promotional purposes, creating new collective works, for resale or redistribution to servers or lists, or reuse of any copyrighted component of this work in other works. Roman Sotner, Jan Jerabek, Ondrej Domansky, Norbert Herencsar, Aslihan Kartci, Jan Dvořák "Practical Design of Fractional-Order Oscillator Employing Simple Resonator and Negative Resistor ", Proceedings of 10th International Congress on Ultra Modern Telecommunications and Control Systems - ICUMT 2018, pp. $\quad$ 1-4, $2018 . \quad$ DOI: 10.1109/ICUMT.2018.8631226. Final version is available at https://ieeexplore.ieee.org/document/8631226 


\title{
Practical Design of Fractional-Order Oscillator Employing Simple Resonator and Negative Resistor
}

\author{
Roman Sotner, Jan Jerabek, Ondrej Domansky, Norbert Herencsar, Aslihan Kartci, Jan Dvořák \\ SIX Research Center \\ Brno University of Technology \\ Brno, Czech Republic \\ sotner@feec.vutbr.cz
}

\begin{abstract}
This contribution presents straightforward design of a fractional-order oscillator employing novel simple impedance inverter (implementing differential voltage current conveyor transconductance amplifier as active element) used for construction of parallel $L C$ resonator and requiring also negative resistor. Design supposing two output waveforms with constant amplitude ratio and phase shift 155 degrees (-25 degrees) supposes two identical constant phase elements (fractional-order capacitors). The key advantages of our solution, stability of ratio of output levels and phase shift between generated waveforms, were confirmed by simulations.
\end{abstract}

Keywords-constant phase element; fractional-order; impedance inverter; oscillator; phase shift; resonator

\section{INTRODUCTION}

The sinusoidal oscillators using standard integer-order passive elements suffer from certain disadvantages and limitations such as providing phase shifts in integer-order portions of $\pi$ only [1]. The design of the oscillator generating arbitrary value of the phase shift between output waveforms supposes implementation of so-called fractional-order passive elements [2]. Recently reported solutions of the fractionalorder oscillators are not providing simple tools for fast design because of complex mathematical description [3]-[6] and are not studying relations between generated levels as well as phase shifts when oscillation frequency tuned [5], [6]. Therefore, we prepared very simple example of the fractionalorder oscillator design using two identical (for simplicity) constant phase elements (fractional-order capacitors) [7], [8], simple electronically controllable impedance inverter and negative resistor. This example uses straightforward approach without necessity of complex expressions and shows simple and easily to be followed design steps. Advantages of presented approach are: a) simple and practically feasible design approach in comparison to complex mathematical ways [3]-[6], b) constant amplitude ratio and phase shift during the tuning process, c) simply controllable condition of oscillation, d) very narrow change of value of parameters used for driving of oscillation frequency leads to sufficient tunability range, e) device implementable by commercially available active elements. Verification of the most important features in PSpice simulations accompany the design described bellow.

Research described in this paper was financed by the National Sustainability Program under grant LO1401 and by the Ministry of Education, Youth and Sports under grant LTC18022 of Inter-Cost program. For the research, infrastructure of the SIX Center was used.

\section{DEFINITION OF ACTIVE ELEMENT USED IN DESIGN}

The modern active elements (AEs) [9] cover features useful for synthesis of various electronic circuits, especially because of their multi-terminal relations and possibility of multiparametric electronic control. The differential voltage current conveyor transconductance amplifier (DVCCTA) represents interesting device allowing useful features for our intentions [9]. We intend to define new behavioral model based on commercially available devices. The internal concept, representing behavior of DVCCTA, is given in Fig. 1. The structure includes differential difference amplifier (DDA) [10] and two current conveyors of second generation (CCII+) [9], [10]. The ideal operation (omitting internal parasitic properties) of the DVCCTA can be explained by the following expressions: $V_{\mathrm{X}}=V_{\mathrm{Y} 1}-V_{\mathrm{Y} 2}, I_{\mathrm{Z}}=I_{\mathrm{X}}, I_{\mathrm{o}}=V_{\mathrm{Z}} \cdot g_{\mathrm{m}}$. The second CCII+ serves actually as operational transconductance amplifier (OTA). Note that adjustable $g_{\mathrm{m}}$ and $R_{\mathrm{X}}$ parameters can be controlled by the standard way (bias current of DC voltage). The same trend of control can be easily obtained for our final implementation, tested with commercially available devices, where external resistance values are varied instead of intrinsic parameters (as shown in Fig. 1).

DVCCTA

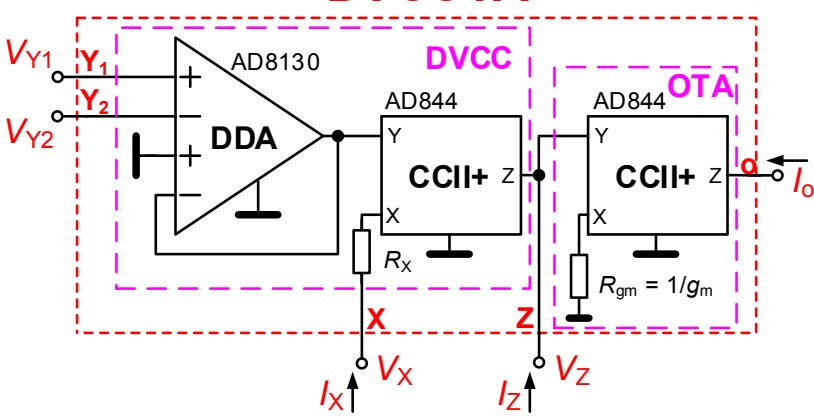

Fig. 1. Proposed concept (principle) and behavioral model of DVCCTA element for further experimental purposes.

\section{IMPEDANCE INVERTER USING DVCCTA}

The topology of designed impedance inverter is shown in Fig. 2. The inverter has the following ideal form of input impedance when general load $Z_{\mathrm{L}}(\mathrm{s})$ is connected: 


$$
Z_{\text {in }}(s)=\frac{1}{2} \cdot \frac{R_{X}}{g_{m}} \cdot \frac{1}{Z_{L}(s)},
$$

Note that this formula is valid also when terminals are interchanged $\left(Z_{\text {in }} \leftrightarrow Z_{\mathrm{L}}\right)$.

\section{Design of the Constant Phase Element}

As an example, we decided to design the oscillator with phase shift (angle) between generated waveforms equal to $\varphi=155^{\circ}\left(-25^{\circ}\right)$. The selected phase shift represents only arbitrary example indicating how this approach can be useful for modeling of behavior for many natural systems [2]. The following constant phase element $(\mathrm{CPE})$ is required for our design. Validity of approximation of $\mathrm{CPE}$ is limited to 4 decades (from $10 \mathrm{~Hz}$ up to $100 \mathrm{kHz}$ ) and phase error is
$\Delta \varphi= \pm 0.5^{\circ}$ in theory. It results into fractional-order capacitor with order $\alpha=5 / 18$ and value $C_{\alpha}=111 \mu \mathrm{F} / \mathrm{sec}^{13 / 18}$ $\left(C_{\alpha}=1 /\left(\omega^{\alpha}\left|Z_{\mathrm{C} \alpha}\right|\right)\left[\mathrm{F} / \mathrm{sec}^{1-\alpha}\right] ;\left|Z_{\mathrm{C} \alpha}\right|=5.407 \mathrm{k} \Omega @ 1 \mathrm{~Hz}\right)$. Figure 3 shows the CPE chain of RC sections having values calculated by method from Valsa et al. [7]. The impedance plot (magnitude and phase) of the CPE are shown in Fig. 4.

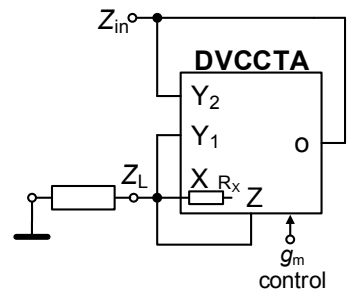

Fig. 2. New topology of the impedance converter based on DVCCTA.

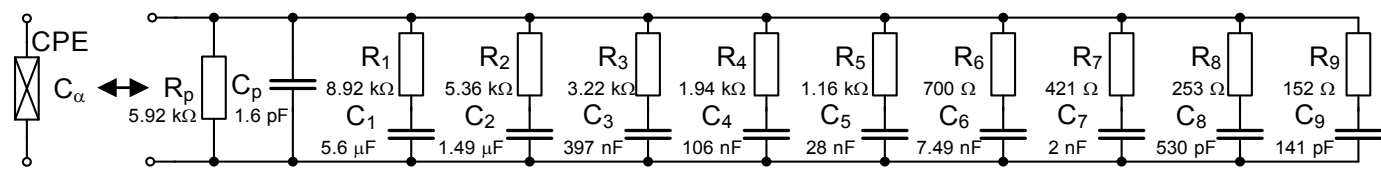

Fig. 3. Designed RC chain of CPE $\left(\alpha=5 / 18, C_{\alpha}=111 \mu \mathrm{F} / \sec ^{13 / 18}\right)$ based on method developed in [7].

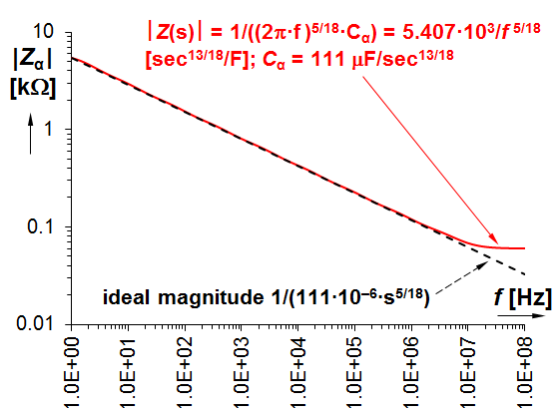

a)

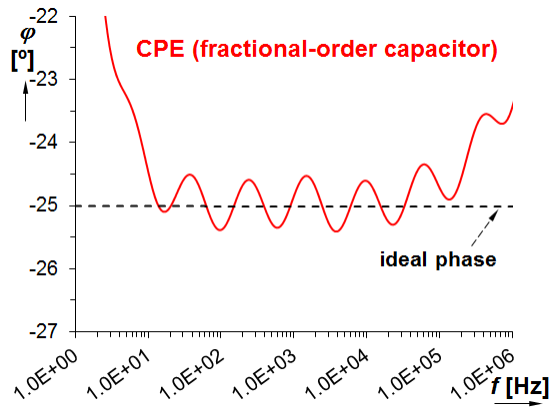

b)

Fig. 4. Impedance plot of designed CPE $\left(\alpha=5 / 18, C_{\alpha}=111 \mu \mathrm{F} / \mathrm{sec}^{13 / 18}\right)$ : a) magnitude response, b) phase response.

\section{FRACTIONAL-ORDER RESONATOR}

The resonator (in the meaning of parallel LC network) represents the important part of the oscillator design [1]. Several fractional-order resonators has been introduced quite recently, [11] for instance based on opamps in Antoniou impedance inverter [11], [12] (many floating passive elements, complicated electronic controllability) and employs combination of fractional- and integer-order capacitors. However, implementation of such resonator in the oscillator design is not necessary as shown in our contribution.

The resonator circuit uses our impedance inverter (described in previous section) and two CPEs (identical $\alpha$ for the simplicity of our example) as shown in Fig. 5. The input impedance of the resonator can be expressed as:

$$
Z_{R}(s)=\frac{s^{\alpha} L_{\alpha}}{s^{2 \alpha} L_{\alpha} C_{\alpha}+1},
$$

where $L_{\alpha}(1)$ represents fractional-order inductance:

$$
L_{\alpha}=\frac{1}{2} \cdot \frac{R_{X}}{g_{m}} \cdot C_{\alpha},
$$

(numerically $L_{\alpha} \cong 55.5 \mathrm{sec}^{23 / 18} / \mathrm{F}$ ) obtained from transformation of $C_{\alpha}$ by the impedance inverter where parameters for setting $L_{\alpha}$ have values $R_{\mathrm{X}}=1 \mathrm{k} \Omega$ and $g_{\mathrm{m}}=1 \mathrm{mS}$. The denominator of (2) has complex root:

$$
p=\left(-\frac{1}{C_{\alpha} L_{\alpha}}\right)^{\frac{1}{2 \alpha}} \exp \left(-\frac{\pi}{2 \alpha} j\right) .
$$

It results into pole in Cartesian form:

$$
p=\left(-\frac{1}{L_{\alpha} C_{\alpha}}\right)^{\frac{1}{2 \alpha}}\left[\cos \left(\alpha \frac{\pi}{2}\right)+j \sin \left(\alpha \frac{\pi}{2}\right)\right],
$$


and value of the pole frequency can be calculated from $\omega_{\mathrm{p}}=(\operatorname{Real}(\mathrm{p})+\operatorname{Imag}(\mathrm{p}))^{-1 / 2}$. The $\alpha$ value is restricted to $0<|\alpha| \leq 1$ and $\varphi_{\max }= \pm 90^{\circ}$. Previously discussed values of $L_{\alpha}$ and $C_{\alpha}$ numerically yield into $p=7.7 \cdot 10^{3}+5.610^{3} j$ that results to $\omega_{\mathrm{p}}=9.52 \mathrm{krad} \cdot \mathrm{s}^{-1}\left(f_{\mathrm{p}}=1.515 \mathrm{kHz}\right)$.

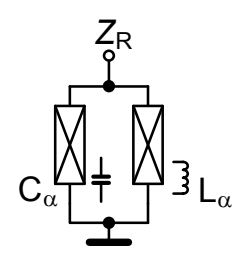

a)

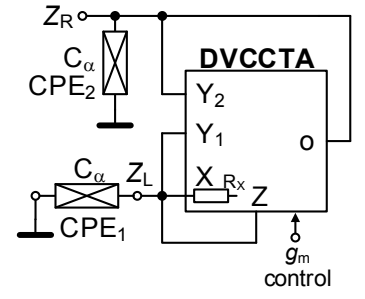

b)
Fig. 5. The fractional resonator network: a) principal topology, b) new circuit solution with impedance convertor based on DVCCTA element.

The frequency $\omega_{\mathrm{p}}$ can be calculated also as:

$$
\omega_{p} \cong\left(\frac{2 g_{m}}{R_{X} C_{\alpha}^{2}}\right)^{\frac{1}{2 \alpha}}=\left(\frac{2}{R_{X} R_{g m} C_{\alpha}^{2}}\right)^{\frac{1}{2 \alpha}}
$$

Magnitude value at the pole frequency can be found from:

$$
\left|Z_{R \max }(s)\right|=\left|Z_{R}\left(\omega \rightarrow \omega_{p}\right)\right|=\left|j^{\alpha} \sqrt{\frac{L_{\alpha}}{C_{\alpha}}} /\left(j^{2 \alpha}+1\right)\right| .
$$

Simultaneous adjustment of $R_{\mathrm{X}}=1 / g_{\mathrm{m}}$ in arbitrarily selected values $\quad 700 \Omega, \quad 1 \mathrm{k} \Omega \quad$ and $1.2 \mathrm{k} \Omega$ yields $Z_{\mathrm{Rmax}}(\mathrm{s})=\left|Z_{\mathrm{R}}\left(\omega \rightarrow \omega_{\mathrm{p}}\right)\right|=468 \Omega, 390 \Omega, 273 \Omega$, respectively, at frequencies of maximal impedance $f_{\mathrm{p}}=5.47 \mathrm{kHz}, 1.515 \mathrm{kHz}$ and $0.79 \mathrm{kHz}$, respectively. Results of the analysis of the circuit shown in Fig. 5 (active devices represented as shown in Fig. 1) are presented in Fig. 6.

\section{OSCILLATOR DESIGN}

The circuit in Fig. 5 can be easily extended to the oscillator when negative resistor, implemented by variable gain amplifier (VGA, implemented by VCA810 chip) and resistor, is added to the node of $Z_{\mathrm{R}}(\mathrm{s})$ as shown in Fig. 7. Standard oscillator design requires analysis of the characteristic equation. However, solution of roots for non-integer-order case is not easily feasible in practice without appropriate software (Matlab) and resulting expressions are uncomfortable for immediate usage ([3]-[6] for example). Fortunately, the circuit in Fig. 7 can be set to oscillations easily by equality of negative resistance value $\left(R_{\text {neg }}=R /(1-A)\right)$ to $Z_{\mathrm{Rmax}}$ at frequency $f_{\mathrm{p}}$ of the resonator (see Fig. 6). It means that condition for oscillation (CO) is given as $R_{\text {neg }} \geq Z_{\mathrm{Rmax}}$ where $R_{\text {neg }} \geq R /\left(1-10^{2(\text { Vset_A-1) }}\right)$, see datasheet of VCA810. This CO can be rewritten as:

$$
R_{n e g} \geq\left|j^{\alpha} \sqrt{\frac{L_{\alpha}}{C_{\alpha}}} /\left(j^{2 \alpha}+1\right)\right|
$$

The gain of the amplifier controlling $\mathrm{CO}$ is expressed as $A \geq 1+R / Z_{\mathrm{Rmax}}$ that leads into practically useful parameter (DC control voltage) setting actually $\mathrm{CO}$ by:

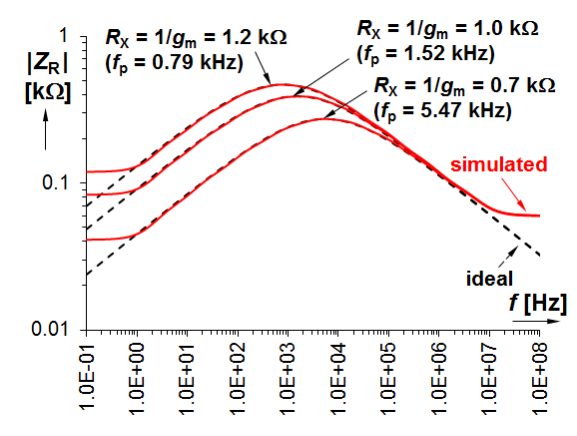

a)

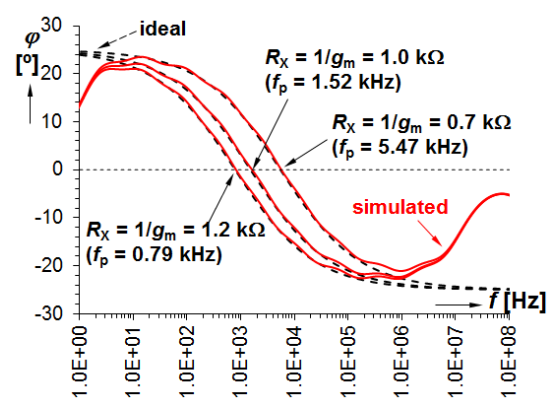

b)

Fig. 6. Impedance plots of the resonator (Fig. 5) for stepping of $R_{\mathrm{X}}=1 / g_{\mathrm{m}}$ : a) magnitude responses, b) phase responses.

$$
V_{\text {set_A }} \geq \frac{1}{2} \log \left(1+\frac{R}{Z_{R \max }}\right)+1 .
$$

The oscillation frequency of the circuit is determined by the resonator frequency (6). The relation between produced waveforms (supposing identical CPEs) can be expressed as:

$$
\frac{V_{1}}{V_{2}}=\left.\frac{-2}{s^{\alpha} C_{\alpha} R_{X}}\right|_{s^{\alpha}=\left(j \omega_{0}\right)^{\alpha}}=-\frac{1}{j^{\alpha}} \sqrt{\frac{2}{R_{X} g_{m}}} .
$$

The formula (9) can be modified into:

$$
\frac{V_{1}}{V_{2}}=\sqrt{\frac{2}{R_{X} g_{m}}} \exp \left(-\alpha \frac{\pi}{2} j\right) .
$$

Analysis of (10) yields to theoretically constant ratio of generated amplitudes and phase shift when frequency is tuned by simultaneous change of $R_{\mathrm{X}}$ and $g_{\mathrm{m}}$.

The numerical values of our design (after substitution to previous equations) are adopted from the resonator design and $R=1 \mathrm{k} \Omega$ (resistor in the negative resistance simulator, Fig. 7). Then, parameters for selected oscillation frequency $f_{0}=1 \mathrm{kHz}$ can be calculated from rearranged equation (6) as $R_{\mathrm{X}}=1 / g_{\mathrm{m}}=\sqrt{ } 2 /\left(\omega_{0}{ }^{\alpha} \cdot C_{\alpha}\right)=\sqrt{ } 2 /\left(\omega_{0}{ }^{5 / 18} \cdot C_{5 / 18}\right)$. It results into $R_{\mathrm{X}}=1 / g_{\mathrm{m}}=1.12 \mathrm{k} \Omega$. The substitution of real values into (9) and (10) results to amplitude ratio $V_{1} / V_{2}=\sqrt{2}$ and phase shift $155^{\circ}\left(-25^{\circ}\right.$ respectively), theoretically. Results of simulations in time domain are shown in Fig. 8 where simulated $f_{0}=1.05 \mathrm{kHz}$ was obtained (and total harmonic distortion about 
$2 \%$ was achieved). Figure 9 indicates the dependence of $f_{0}$ on $R_{\mathrm{X}}=1 / g_{\mathrm{m}}$ tested in range $0.7 \rightarrow 1.2 \mathrm{k} \Omega$ leading to $f_{0}$ range from $0.82 \mathrm{kHz}$ up to $5.72 \mathrm{kHz}$ (ideal range $0.79 \rightarrow 5.47 \mathrm{kHz}$ ). The harmonic distortion varied from 0.5 up to $2.5 \%$. Expected dependences of phase shift between generated waveforms and their amplitude ratios are shown in Fig. 10 for particular settings. For tuning of $f_{0}$ amplitude stabilization (controlling $A$ by $V_{\text {set_A }}$ automatically), i.e. automatic gain control (AGC) system, has been used (not shown due to space restrictions).

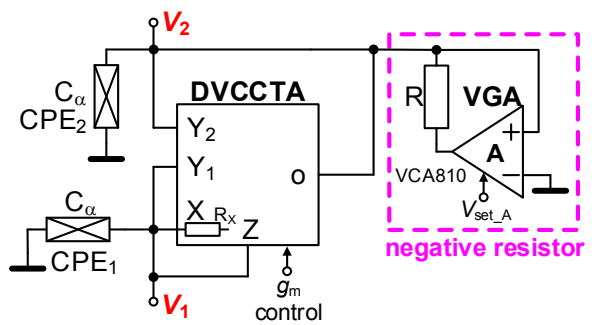

Fig. 7. Proposed resonator applied to the design of oscillator.

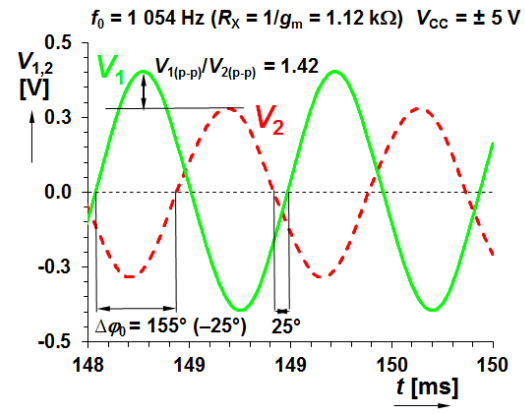

Fig. 8. Generated output waveforms $\left(f_{0}=1.05 \mathrm{kHz}\right)$.

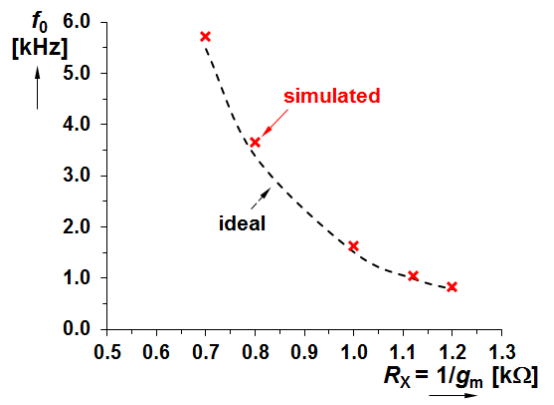

Fig. 9. Control of $f_{0}$ by value of $R_{\mathrm{X}}=1 / g_{\mathrm{m}}$.

\section{CONCLUSION}

This paper presents practical approach to design of the fractional-order oscillator based on two identical fractionalorder passive elements employing special active device (differential voltage current conveyor transconductance amplifier) applied in very simple resonator. Operation of the circuit confirming intended phase shift $155^{\circ}\left(-25^{\circ}\right)$ between generated amplitudes, not available by the standard integerorder solution, has been verified by PSpice simulations at oscillation frequency $1 \mathrm{kHz}$ and even for frequency tuning between $0.82 \mathrm{kHz}$ and $5.72 \mathrm{kHz}$. The most important advantage of our solution is stability of value of phase shift and ratio of amplitudes during tuning of $f_{0}$.

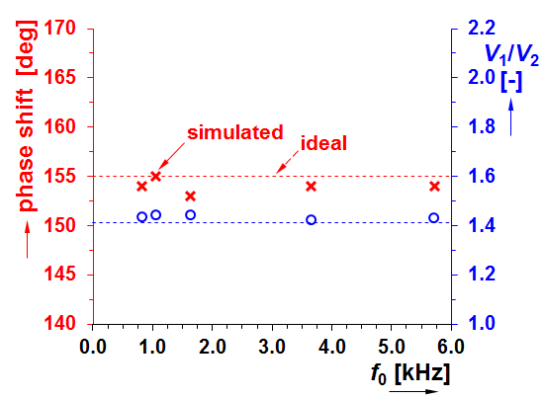

Fig. 10. Dependence of phase shift and amplitude ratio on tuning of $f_{0}$.

\section{REFERENCES}

[1] R. Senani, D. R. Bhaskar, V. K. Singh, R. K. Sharma, Sinusoidal Oscillators and Waveform Generators using Modern Electronic Circuit Building Blocks, Springer, Switzerland, 2016.

[2] A. S. Elwakil, "Fractional-order circuits and systems: An emerging interdisciplinary research area," IEEE Circuits and Systems Magazine, vol. 10, no. 4, pp. 40-50, 2010.

[3] A.G. Radwan, A.S. Elwakil, A.M. Soliman, "Fractional-order sinusoidal oscillators: design procedure and practical examples," IEEE Trans. on Circuits and Systems I: Regular Papers, vol. 55, pp. 2051-2063, 2008.

[4] L.A. Said, A.G. Radwan, A.H. Madian, A.M. Soliman, "Two-port two impedances fractional order oscillators," Microelectronics Journal, vol. 55, pp. 40-52, 2016

[5] A. Kartci et al., "Fractional-order oscillator design using unity-gain voltage buffers and OTAs," in Proc. of 2017 IEEE 60th International Midwest Symposium on Circuits and Systems (MWSCAS), Boston, MA, 2017, pp. 555-558.

[6] A. Kartci, N. Herencsar, J. Koton and C. Psychalinos, "Compact MOSRC voltage-mode fractional-order oscillator design," in Proc. of European Conference on Circuit Theory and Design (ECCTD), Catania, 2017, pp. 1-4

[7] J. Valsa, J. Vlach, "RC models of a constant phase element," International Journal of Circuit Theory and Applications, vol. 41, no. 1, pp. 59-67, 2013

[8] G. Tsirimokou, C. Psychalinos, A. S. Elwakil, "Emulation of a Constant Phase Element Using Operational Transconductance Amplifiers," Analog Integrated Circuits and Signal Processing, vol. 85. no. 3, pp. 413-423, 2015.

[9] R. Senani, D. R. Bhaskar, A. K. Singh, Current Conveyors: Variants, Applications and Hardware Implementations, Springer, Berlin, Germany, 2015.

[10] D. Biolek, R. Senani, V. Biolkova, Z. Kolka, "Active elements for analog signal processing: Classification, Review and New Proposals," Radioengineering, vol. 17, no. 4, pp. 15-32, 2008

[11] A. Adhikary, S. Sen, K. Biswas, "Practical Realization of Tunable Fractional Order Parallel Resonator and Fractional Order Filters," IEEE Transactions on Circuits and Systems I: Regular Papers, vol. 63, no. 8, pp. 1142-1151, 2016.

[12] A. Adhikary, S. Choudhary, S. Sen, "Optimal Design for Realizing a Grounded Fractional Order Inductor Using GIC," IEEE Transactions on Circuits and Systems I: Regular Papers, vol. 65, no. 8, pp. 2411-2421, 2018 . 